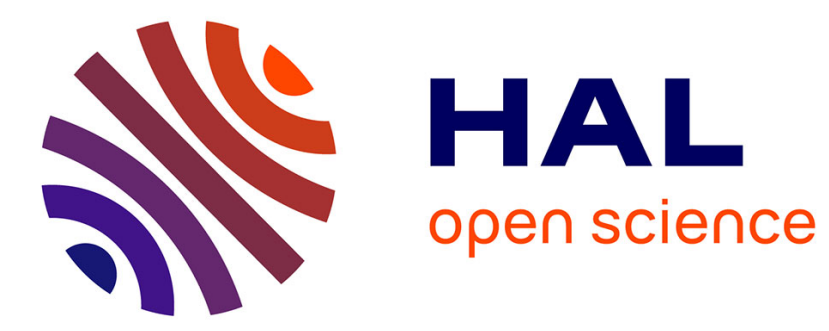

\title{
Le désastre de Montaperti chez Guittone d'Arezzo
}

Cécile Le Lay

\section{To cite this version:}

Cécile Le Lay. Le désastre de Montaperti chez Guittone d'Arezzo. Arzanà. Cahiers de littérature médiévale italienne, 2005, 11, pp.17-45. halshs-00394135

\section{HAL Id: halshs-00394135 https://shs.hal.science/halshs-00394135}

Submitted on 14 Jun 2017

HAL is a multi-disciplinary open access archive for the deposit and dissemination of scientific research documents, whether they are published or not. The documents may come from teaching and research institutions in France or abroad, or from public or private research centers.
L'archive ouverte pluridisciplinaire HAL, est destinée au dépôt et à la diffusion de documents scientifiques de niveau recherche, publiés ou non, émanant des établissements d'enseignement et de recherche français ou étrangers, des laboratoires publics ou privés. 


\section{Le désastre de Montaperti chez Guittone d'Arezzo}

Cécile Le Lay

\section{Citer ce document / Cite this document :}

Le Lay Cécile. Le désastre de Montaperti chez Guittone d'Arezzo. In: Arzanà 11, 2005. La poésie politique dans I'Italie médiévale. pp. 17-45;

doi : 10.3406/arzan.2005.946

http://www.persee.fr/doc/arzan_1243-3616_2005_num_11_1_946

Document généré le 27/04/2017 


\section{Le désastre de Montaperti chez Guittone d'Arezzo}

Ta vibrante émotion qui émane de la chanson Ahi lasso, Lor è stagion de doler tanto (Hélas! Le temps du grand deuil est venu, XIX) ${ }^{1}$ ne semble laisser aucun doute sur le fait qu'elle a été composée peu de temps après la cuisante défaite des guelfes à Montaperti (4 septembre 1260). Mais la spontanéité apparente de cette réaction plus ou moins immédiate ne peut nous dispenser de poser la question des modeles et du genre dans lequel s'inscrit cette composition, surtout pour un auteur comme Guittone, si fin connaisseur des enjeux littéraires de son époque ${ }^{2}$.

Dès l'incipit, le poète choisit de se rattacher à la tradition du planh en cherchant à tirer un enseignement moral de cette catastrophe. L'impact, voire la valeur paradigmatique qu'acquiert aussitôt une telle démarche sont attestés par la chanson de Chiaro Davanzati ( $A$ hi dolze e gaia terra fiorentina: Hélas! Douce et gaie ville de Florence, $\mathrm{XXV}^{3}$ ), écrite quelques années plus tard, qui se réfere ostensiblement au lexique et à la thématique du modèle guittonien, mais pour les adapter à la déploration de la défaite gibeline de Bénévent (1266). Il s'agira donc de comprendre comment l'actualité politique redevient en Italie matière poétique, après l'absence d'intérêt apparent des poètes siciliens pour un sujet d'inspiration pourtant présent chez leurs modèles provençaux. De fait, un tel choix permet à Guittone de renouer avec la tradition en langue d'oc des chansons politiques (cf. la Chanson de la croisade albigeoise de Guillaume de 
Tudèle, ou d'autres compositions plus satiriques comme les sirventès), et d'inaugurer en langue italienne un genre destiné à un avenir glorieux (grâce à Dante et à Pétrarque, pour ne citer que les auteurs les plus célèbres). Comme nous le verrons, Guittone transpose sur un plan poétique le thème très répandu à son époque de Florence "nouvelle Rome ", à l'image de la translatio imperii consacrée par la littérature française (par exemple dans Cligès de Chrétien de Troyes, qu'il connaissait bien). Par ailleurs, l'étude du terme ragione permettra d'établir certains rapprochements avec la pensée "politique" (au sens de la polis, la cité) de l'époque (on songe à l'importance des textes et des idées de Brunetro Latini ${ }^{4}$ ).

Afin de mieux saisir la portée de ces vers, commençons par en rappeler brièvement le contexte historiques. Soulignons avant tout - et Guittone le fait très bien ressortir - que les enjeux de la bataille de Montaperti débordent largement le cadre d'un conflit entre deux communes rivales telles que Florence et Sienne : en effet, elle s'inscrit dans la lutte que Manfred, roi de Sicile, a entreprise pour consolider l'héritage de Frédéric II (†1250) dans la péninsule italienne, et elle marque un coup d'arrêt brutal à la phase d'expansion spectaculaire (voir la deuxième strophe) que la commune florentine connaissait depuis que les guelfes avaient pris le pouvoir après la mort de l'empereur, en associant la haute bourgeoisie des affaires (popolo grasso) au gouvernement de la ville.

C'était en effet l'époque où la commune de Florence avait décidé de battre le florin d'or (en 1252), futur " dollar du Moyen Âge", usurpant ainsi le privilège que l'empereur Frédéric II s'était réservé pour toute frappe de monnaie d'or. De plus, après sa victoirc sur les gibelins, le peuple florentin avait choisi le lion (Marzocco) comme emblème de son pou- 
voir (c'est le mot-charnière entre la deuxième et la troisième strophe), et la ville décida même de tenir un vrai lion en cage près du Baptistère, montrant ainsi sa puissance à l'instar de l'empereur qui avait coutume d'exhiber dans sa suite des fauves exotiques. Par ailleurs, la construction du palais du Peuple, commencée en 1255, révélait aussi dans ses inscriptions l'image de domination que Florence voulait reproduire : celle de la Rome antique, dont les victoires incessantes avaient élargi le pouvoir à l'ensemble du monde connu, tout en assurant le maintien de la justice et de la paix ${ }^{6}$.

Après une dizaine d'années où la suprématie du peuple florentin s'était affirmée de façon incontestée, la déroute des armées florentines aux portes de Sienne fut perçue comme un véritable cataclysme. Elles avaient pourtant déjà subi un premier revers la même année, le 18 mai 1260 à Santa Petronilla, face aux cavaliers allemands que le roi Manfred avait enfin accepté d'envoyer en renfort à Sienne, sur les instances répétées de la délégation des gibelins florentins exilés dirigée par Farinata degli Uberti (celui qui allait ensuite s'opposer à la destruction totale de Florence après l'anéantissement des guelfes : Dante immortalisera le souvenir de ce singulier personnage grâce au chant $X$ de l'Enfer).

Comme chaque année à l'approche de l'hiver, Florence se chargeait du ravitaillement de Montalcino, forteresse alliée située au sud de Sienne, mais cetre fois-ci les dirigeants ne tinrent pas compte des conseils de prudence que certains chevaliers guelfes avaient donnés : ils comptaient profiter de cette occasion pour faire oublier la récente défaite en passant sous les remparts de leurs ennemis avec toute l'armée, guidée par le fameux char décoré de quatre lions (Carroccio) qui transportait les gonfalons, un autel et la cloche (Martinella) qui avait sonné pendant un mois jour et nuit pour annoncer les prépa- 


\section{CECILE LE LAY}

ratifs de guerre. On a calculé qu'il y avait environ 70000 hommes prêts à combattre (dont 3000 cavaliers), y compris les contingents guelfes envoyés en renfort par Lucques, Pistoia, Prato, Arezzo, Bologne, Pérouse, et autres. L'armée était suivie d'une colonne de ravitaillement composée de 20000 bêtes de somme chargées de vivres, en partie destinés à Montalcino (voir à la quatrième strophe l'énumération du butin rassemblé par les vainqueurs).

Les Florentins exilés attendaient depuis longtemps cette occasion pour prendre leur revanche : malgré la paix signée entre les partis en janvier 1251, la plupart d'entre eux avaient en effet été chassés hors de Florence en août de la même année. Depuis Sienne, Farinata et ses alliés avaient donc soigneusement préparé le terrain en obtenant le renfort de 800 cavaliers et, surtout, en préparant un piège : sous couvert de secret, ils avaient fait croire à certains représentants du peuple qu'au moment venu des traîtres ouvriraient les portes de Sienne pour livrer la ville aux assaillants, alors qu'ils s'étaient déjà entendus avec les gibelins restés dans l'armée florentine pour qu'ils changent de camp pendant la bataille (en remplaçant la croix rouge de leur armure par la croix blanche des exilés).

La confusion provoquée par cetre trahison fut indescriptible et l'on raconte, comme Dante le rappellera aux vers 85 et 86 du même chant $X$ de l'Enfer, que " le grand carnage et l'hécatombe// [...] teignirent en rouge l'eau de l'Arge "7 (l'Arbia, la rivière qui passait non loin du champ de bataille). Sienne ramena environ 20000 prisonniers dans ses murs, dont près de la moitié mourut de privations au cours des dix années qui suivirent.

Sur un plan métrique, la chanson est composée de six strophes capfinidas et d'un envoi adressé ironiquement aux barons de toute l'Italie. Chaque strophe a deux pieds symé- 


\section{LE DESASTRE DE MONTAPERTI}

triques ( $\mathrm{ABBA}, \mathrm{CDDC}$ ), et une sirma dont la structure métrique (EFGgFfE) est reprise dans le congé, à l'imitation de la tornada provençale. Comme l'incipit l'annonce, il s'agit d'abord d'un planh : le réseau sémantique des regrets, des pleurs et de la mort est bien visible dans la première partie de la chanson. Puis, à partir de l'apparition du mot "fou " ("folle ") qui marque le passage entre la quatrième et la cinquième strophe, les regrets se transforment en sarcasmes : la folie semble alors résumer tout ce que le poète reproche aux gibelins florentins.

La transposition sur un plan poétique d'un événement marqué par un enjeu aussi important devait amener le poète à en proposer une image particulièrement saisissante. Guittone choisit ainsi de se lamenter sur le sort de Florence en donnant vie à ses deux emblèmes (la fleur de lys et le Marzocco). Au vers 5 ("la noble Fleur de tous temps porteuse de fruits": "l'alta Fior sempre granata"), le mot fiore, tiré de l'ancien nom Fiorenza, est féminisé comme en ancien français et en provençal (contrairement à l'italien pour lequel il reste masculin comme en latin), puis associé au terme "granata " qui fait référence à la tradition poétique provençale (granar signifie "donner du fruit " et fut également repris par les Siciliens). Par le biais de cette première personnification-métaphore, Guittone revient au planh lyrique pour la femme aimée, tout en détournant son contenu habituel : l'intensité dramatique de la catastrophe est condensée dans cette image de noblesse et de prospérité sur laquelle pèse une menace de mort. Comme le fait remarquer Ruggero Stefanini, cette chanson montre que le poète se sent fortement impliqué dans une situation politique encore en pleine évolution dans laquelle il espère pouvoir intervenir ("si on ne la secourt pas au plus tôt", v. 8$)^{8}$. Le premier vers de la deuxième strophe se termine par 


\section{Cécile Le lay}

l'expression lapidaire "Fleur défleurie" (" sfiorata Fiore", v. 16), derivatio privative qui désigne l'anéantissement brutal de la ville et annonce l'opposition entre la grandeur des mérites décrits dans cette strophe et le reste du poème : comme pour la mort de la femme aimée, la douleur du présent incitera à exalter les vertus passées. La figure du lion, glorifié puis renversé à la charnière entre la deuxième et la troisième strophe, souligne la violence de la chute. La troisième occurrence du mot fiore apparaît dans le congé au vers 93, "Florence, fleur qui toujours se renouvelle" ("Fiorenza, fior che sempre rinovella"), illustrant le dernier avatar de cette fleur qui ne reprend vie que pour être cruellement tournée en dérision. La triple occurrence de cette métaphore filée reprend ainsi le triple mouvement logique et rhétorique de la chanson : exaltation et avilissement mis en opposition, suivis d'une parodie de triomphe. L'effet de choc de l'antithèse initiale fait jaillir avec d'autant plus de violence les sarcasmes du retournement final, au moment de la brève réapparition de la fleur aux derniers vers.

La Lettre XIV, écrite par Guittone pour la même circonstance, ne reprend cette image de la Fleur qu'à travers l'adjectif dérivé disfiorati qui désigne de façon récurrente les Florentins", mais nous la retrouvons plusieurs fois dans la chanson déjà citée de Chiaro Davanzati ${ }^{10}$, et même dans le De vulgari eloquentia, où Dante y fait allusion en donnant un exemple de "construction savoureuse et gracieuse, mais aussi sublime, qui est le propre des écrivains les plus illustres ", alors qu'il dénigrera explicitement Guittone à la fin du passage $^{11}$.

Dès le deuxième vers, le poète regroupe sous une même expression hautement symbolique tous ceux qui sont concernés par le deuil : il s'agit de "ciascun om che ben ama 
Ragione", que l'on peut traduire par "tous ceux qui sont épris du bon droit ", sachant que le terme ragione couvre un ensemble de notions voisines telles que raison, sagesse, justice, en plus du sens spécifique de droit (écrit ou autre). C'est en effet un terme clef de la pensée politique de l'époque dans la mesure où il participe de ses trois composantes: éthique et/ou morale (si l'on veut distinguer morale collective et individuelle), juridique et politique ${ }^{12}$. Ce n'est donc pas avant tout un parti politique qu'il défend ou qu'il accuse ici, mais la notion même de Ragione, mise en évidence dès le début du poème. A-t-il lui-même toujours été guelfe ? Claude Margueron préfere ne pas trancher en rappelant " que les positions n'étaient pas prises une fois pour toutes et que la société italienne du $\mathrm{XIII}^{\mathrm{e}}$ siècle ignorait les cloisons étanches entre factions " ${ }^{13}$. Cependant, Guittone ne se limite pas à bâtir un discours moral détaché de toute réalité historique. Par la suite (troisième strophe), le poète précise en effet que les gibelins florentins sont condamnables du fait qu'ils ont plusieurs fois trahi leur ville. Notons au passage que changer de camp pendant une bataille signifie rompre le serment écrit et lu publiquement dans une église avant le début des hostilités : il s'agit donc bien de défendre le droit et la justice, comme l'évoquait le mot ragione. Ce sens est renforcé ensuite a contrario par l'accusation de folie portée contre les traîtres. Il est spécifié plus loin que c'est la disparition à Florence de " ses anciens us romains dignes d'honneur" ("l'onorato antico uso romano ", v. 6) qui est la cause de son malheur. Au nom des origines romaines que la ville aime à proclamer ${ }^{14}$, Guittone lui reproche d'avoir trahi le modèle politique et juridique qu'elle s'était forgé : en effet, elle est en train de perdre la richesse de son illustre héritage, "sa grandeur riche et honorée " ("l'onorata sua ricca grandezza ", v. 9). 
En s'appuyant sur l'étude des emplois de ragione, on s'aperçoit que c'est l'un des mots-clés de plusieurs autres compositions " politiques" écrites par Guittone avant son entrée chez les chevaliers de la Vierge, les Gaudenti; chaque fois, sa présence révèle l'attention particulière que le poète portait aux fondements indissolublement éthiques et juridiques de la vie en société, c'est-à-dire de la politique. En effet, ces occurrences peuvent être mises en parallèle avec certains passages significatifs des textes qui présentent les développements de la réflexion politique de l'époque, comme ce passage de Brunetto Latini, inspiré de Cicéron :

La cité est une réunion de personne faites pour vivre selon la raison ; de sorte qu'on n'appelle pas citoyens d'une même commune ceux qui seraient rassemblés à l'intérieur d'une seule muraille, mais ceux qui sont rassemblés pour vivre selon une même loi [...]. Et là où il [Cicéron] dit qu'ils ne gardent pas la raison, j'entends par "raison" la justice, celle à propos de laquelle les livres de la loi disent que justice est une volonté d'esprit ferme et permanente qui donne à chacun ce qui lui est $\mathrm{du} \hat{u}^{15}$.

Par exemple, dans un célèbre passage de la chanson de l'exil volontaire (d'Arezzo, sa ville natale), Gente noiosa e villana (Des gens nocifs et vulgaires, $\mathrm{XV})^{16}$, le mot ragione apparaît dans un contexte comparable, où le sens spécifique de droit s'ajoute au sens général de justice ou de sagesse :

La peine me vient aussi au souvenir des bonnes coutumes et du droit (bon uso e ragione) qui en sont partis et du crime, de l'injustice et de la trahison qui y règnent $(X V, 29-32)^{17}$. 


\section{LE DÉsastre de MonTAPERTI}

Ce même terme sera repris de façon significative dans la chanson XXV (Ora parrà s'eo saverò cantare: Nous allons voir si je saurai chanter) ${ }^{18}$, qui marque sa conversion et la rupture avec sa production poétique antérieure : pour écrire de bons vers, nul besoin d'obéir à l'inspiration d'Amour et à sa folie, car le poète entend désormais se faire le chantre de la justice. Ce n'est pas ici le lieu pour intervenir dans les discussions sur le caractère littéraire et moral de cette " conversion", ni sur le rôle effectif que Guittone a pu jouer dans l'agencement bipartite de son œuvre (notamment pour le manuscrit Laurenziano Rediano 9). Qu'il suffise de rappeler que, sur un plan civil et religieux, Guittone est entré dans un ordre qui a pour mission de rétablir et de maintenir la paix et la justice dans les communes selon les vues de l'Église.

La première strophe de la chanson de Montaperti se termine par la question dramatique que le poète adresse à Dieu : "Dieu! Comment as-tu souffert/que la justice périsse et l'iniquité triomphe?" ("Deo, com'hailo sofrito,/deritto pèra e torto entri 'n altezza ?", v. 14-15). La principale préoccupation qui est soulignée ici concerne la disparition de la justice (" deritto pèra") et le triomphe de son contraire ("torto entri "n altezza"), comme dans la chanson $X V$, où le poète déplorait le départ de "bon uso et de ragione", et la présence de " torto" et de "falsezza ". À la suite de Francesco Mazzoni, nous pouvons donc affirmer que l'ample discours grâce auquel le poète justifie son blâme " n'a que l'apparence d'un discours exclusivement moral car il aborde la problématique du bonum commune et de la vie en société " 19 .

La strophe suivante reprend le dernier mot de la première strophe, "altezza", et brosse un tableau de l'expansion économique et politique de la ville, au temps où elle était fidèle 
à elle-même au point d'adopter le style impérial de la Rome antique dans sa façon de conquérir de nombreux territoires par son seul courage. La deuxième occurrence de ragione dans cette chanson apparaît alors dans l'expression "a ragione" (v. 24), qui peut avoir le sens lexicalisé de "à juste titre", mais qui retrouve pleinement son sens juridique grâce au contexte : en effer, ce qui justifiait la prospérité de Florence n'était autre que la conscience qu'elle avait de son devoir de concentrer ses efforts sur le maintien de la justice et de la paix (v. 25-26), de même que Rome assurait l'antique pax romana. Et c'est encore le respect de cet engagement, c'est la fidélité à cet héritage qui ont permis à son emblème héraldique, le Lion, de conquérir une réputation universelle (v. 27 30), à l'image de l'Aigle romaine. Dans toute la deuxième strophe, contrairement à la première, les verbes sont aux temps du passé : l'époque dont il parle est bien révolue, et le poète épanche ainsi ses regrets tout en rappelant la nécessité pour le salut de la res publica florentine de maintenir à tout prix la concorde civile en même temps que le bon fonctionnement de la justice.

Au temps de sa production lyrique, avant même de renier son inspiration amoureuse pour se conformer aux nouvelles convictions religieuses affichées depuis sa conversion, Guittone s'indignait donc déjà face à la corruption et à la perte des valeurs, tout en indiquant un idéal de référence à la fois moral, juridique et politique: la Rome antique. C'est l'exercice de la Raison en premier lieu, emblème de toute une tradition morale et juridique qui remonte à l'Antiquité, qui aurait dû permettre de faire régner la justice et la paix entre les communes. Et même si cette façon de se valoriser en se faisant le défenseur et l'héritier d'un passé mythique n'est pas une nouveauté pour la culture médiévale, Guittone, deve- 
nant en quelque sorte le porte-parole poétique de la réflexion politique menée à son époque, est à l'origine d'une longue tradition de poésie civile en langue vulgaire qui revendique ses titres de noblesse en s'appropriant ce mythe exemplaire.

La troisième strophe commence alors par l'emblème du Lion, qui s'anime soudain, par la prosopopée, pour illustrer tous les malheurs infligés à Florence par une partie de ses enfants, issus de sa noble lignée ("la schiatta gentil sua", v. 36), mais prêts à tout pour obtenir le pouvoir, même à sacrifier leur patrie. Adoptant ici le point de vue des guelfes et du peuple florentin, Guittone continue son récit historique par symboles et retrace les différentes tentatives des gibelins pour s'emparer du pouvoir (tentatives caractérisées par une profonde ingratitude envers leurs concitoyens), avant leur victoire ignominieuse de Montaperti qui marque l'anéantissement spectaculaire du Lion jusque-là invincible : le premier coup mortel (v. 40) avait été porté lors de la première expulsion des guelfes (1248), suivie pourtant par la guérison du Lion et le pardon des coupables (v. 41-42, se référant à la paix signée entre les partis en janvier 1251), mais une nouvelle blessure (v. 43) lui avait été infligée par la conjuration des gibelins (1258) et, malgré ce nouveau coup, le Lion avait montré sa force en leur faisant grâce (v. 44). Pour tout remerciement, les gibelins l'ont à présent abattu, lui et ses membres (v. 45).

La troisième et la quatrième strophe sont liées par le motcharnière " conquis[o] " ("abattu"), qui se réfere d'abord au Lion (" or hanno lui e soie membre conquise", v. 45), puis à ce dont il est l'emblème : " la grande Commune de Florence est abattue" (" conquis'è l'alto Comun fiorentino ", v. 46). Comme le fait remarquer Ruggero Stefanini à propos de la 
poésie entière, l'enchaînement des capfinidas, particulièrement resserrée ici par le lien syntagmatique (" conquise" "conquis'è "), contribue à la cohésion logique et syntaxique du discours $^{20}$. Ce terme constitue aussi l'un des nombreux exemples de transposition du vocabulaire amoureux dans un contexte politique : ce n'est plus l'Amant qui " tombe " sous les coups d'Amour, mais la commune, sous les coups des traîtres qu'elle a nourris. Contrairement à la nécessité que Guittone éprouvera de poser un regard critique sur le lexique amoureux à partir de la chanson XXV (qui marque le tournant de sa conversion), il se contente ici de le resémantiser, en insistant sur l'importance des conséquences historiques (le même terme se retrouvera au dernier vers, pour dénoncer le pseudo-triomphe final).

La quatrième strophe permet au poète de revenir au présent pour prendre du recul par rapport à la simple vision d'une défaite guelfe, aussi déplorable soit-elle. Les conséquences désastreuses d'une telle défaite sur un plan territorial sont décrites avec force détails, de la liste des villes cédées à Sienne au butin de guerre qui comprenait les symboles de la puissance de Florence (la cloche, les drapeaux et les engins de guerre). La catastrophe semble être perçue comme une juste punition, puisque toutes les vexations qu'elle avait fait subir à sa rivale sous les yeux de la péninsule entière lui sont à présent infligées en retour : n'est-ce pas déjà une pointe d'ironie contre les formes d'abus de pouvoir qui se croient à l'abri d'un retournement de la fortune? Cependant, la conclusion de la strophe renouvelle l'accusation portée contre la noblesse gibeline : cette triste engeance est à présent accusée de folie, notion qui sert de transition entre la quatrième et la cinquic̀me strophe, renforcée là aussi par la transformation de la reprise («folle »//« Foll'è ») : 


\section{LE DESASTRE DE MONTAPERTI}

Et tout cela advient à cause

de cette engeance plus que toute autre folle.

Fol est qui fuit son bien et cherche son dommage,

et fait que son honneur se tourne en honte (v. 59-62) 21 .

Le mépris de la paix et de la justice (le bien commun) est en effet signe de déraison : les couples d'antonymes qui se suivent au début de cette strophe dénoncent la confusion entre "bien " et "dommage" ("prode" et "danno ", v. 61), honneur et honte (" onor " et "vergogna", v. 62), amplifiée plus loin par "grand plaisir " et "grand malheur" ("gran piacer" et "gran danno ", v. 64). L'ultime conséquence de cette folie est l'acte le plus irréfléchi (et le plus condamnable ${ }^{22}$ ) qu'il soit possible d'envisager pour un citoyen : la haute trahison, qui entraîne ici au plan politique le renoncement à sa propre liberté, la célèbre Libertas dont s'enorgueillissaient tant les Florentins, ainsi que l'abandon de la ville à un parti politique composé de traîtres (" une seigneurie félonne et mauvaise ", v. 65) et à ses pires ennemis (v. 66), puisque les mercenaires allemands sont accueillis dans ses murs (v. 69-72). Guittone n'hésite pas à soulever ici la question de la légitimité du pouvoir, question devenue brûlante au lendemain de Montaperti. De même, profondément affecté par cette guerre fratricide, Brunetto Latini souligne à différentes reprises qu'en aucun cas il ne voudrait voir sa ville entre les mains d'un seul seigneur ou déchirée par des divisions internes qui ne pourraient que la mener à sa perte : lidéal auquel il se réfere explicitement est pour lui aussi le bien commun, qui devrait permettre d'cuvrer dans la paix ${ }^{23}$.

Dès lors, l'indignation tourne au mépris qui, enjambant la séparation d'avec la dernière strophe, s'exprime par des 


\section{Cécile le Lay}

propos sarcastiques adressés à ceux qui ont pris possession de Florence (de " $\grave{A}$ vous qui êtes à présent à Florence, je dis ", v. 67, jusqu'à la fin de la sixième strophe, v. 90). Parodiant leur folie qui confond liberté et tyrannie ("bona libertà " et "signoria fella e malvagia ", v. 63-64), Guittone les encourage à bien servir les nouveaux occupants qui ont massacré leur famille (v. 69-72) et il déclare même qu'il serait ravi s'ils devaient leur donner de fortes sommes d'argent pour les récompenser de leurs efforts (v. 73-75). La sixième strophe commence par cette idée antiphrastique de récompense bien méritée, mais l'aberration est amplifiée par le poète qui indique clairement qu'il faudrait aussi remercier Guido Novello ( i Conti»), Farinata ( li Uberti»), et tous ceux qui ont mis Sienne en leur pouvoir (v. 77-79). Le propre du sarcasme étant de dénoncer une affirmation en l'exagérant jusqu'à l'absurde, la vérité ne peut qu'éclater au grand jour. En effet, comment peut-on croire avoir remporté la victoire lorsque vos pires ennemis sont dans vos murs! Reprenant presque mot à mot l'énumération des pertes territoriales de la quatrième strophe, Guittone transforme alors toute la fin de la dernière strophe en une marche triomphale de Florence sur l'ensemble de la Toscane (v. 80-90).

Les sarcasmes atteignent leur paroxysme dans le congé où tous les barons de la péninsule sont conviés à la cour de Florence pour fêter son couronnement. L'hyperbole est à la mesure de son indignation :

Barons de Lombardie et de Rome et des Pouilles et de Toscane et de Romagne et des Marches, Florence, fleur qui toujours se renouvelle,

à sa cour vous appelle car elle veut devenir le roi des Toscans 
après que les Allemands

elle les a abattus par la force, et les Siennois (v. 91-97).

Le congé se conclut par un bel exemple d'épiphrase, après une longue série d'apostrophes qui s'apparentent à cette figure de style par une même impression d'ajout spontané. Dans son étude L'epifrasi in Guittone, Paolo Cherchi avance sa propre définition de cette figure très peu étudiée : il s'agit de " deux membres de phrase de même valeur syntaxique qui, habituellement unis, sont séparés par un troisième élément qui est lié à chacun d'eux. Mais la position du second membre est imprévisible et il semble justement avoir été ajouté à une phrase achevée sur le plan syntaxique". Le recours fréquent de Guittone à cette figure de style serait ainsi « le signe le plus évident d'un discours in fieri, [...] le résultat d'une oratio perpetua" ${ }^{24}$. Toutefois, contrairement à ce que semble croire Paolo Cherchi, "la voix du poète et la voix de l'orateur ", qu'il distingue, n'étaient pas clairement dissociables à l'époque : en réalité, Guittone ne fait pas figure d'exception puisqu'en croisant ces deux voix il se situe à l'intérieur d'une longue tradition qui remonte à l'Antiquité ${ }^{25}$.

En définitive, il s'agit bien pour Guittone de confondre par tous les moyens (rhétoriques et stylistiques bien sûr !) les gibelins en leur prouvant que " la victoire dont ils se félicitent de façon stupide n'est, à bien y regarder, qu'une effroyable défaite ", comme le souligne à juste titre Ruggero Stefanini, qui parle même de "provocation du paradoxe " 26 (l'ironie de ses sarcasmes, avons-nous dit, est en quelque sorte la parodie de leur folie aveugle). Il nous semble toutefois excessif (et anachronique) d'interpréter cette chanson comme la preuve que " le guelfe Guittone est en accord avec son temps et donc 
[qu'il] croit fermement en un état régional ", ou bien que "l'unique problème pour Guittone [...] est que la construction d'un état régional toscan pouvait seulement être la tâche de Florence guelfe [...] et non [...] d'une Sienne gibeline ": même s'il est intolérable à ses yeux (et pour bien d'autres) que Florence soit sous la tutelle de Sienne, la prudence de Claude Margueron nous semble préférable ${ }^{27}$.

Mieux vaux insister sur le fait que le fréquent recours de Guittone aux finesses de la rhétorique répond avant tout à un souci d'éloquence, comparable à celui d'un avocat lors d'une plaidoirie. N'oublions pas que Guittone était en relation avec de nombreux personnages influents de l'époque, ainsi qu'avec de nombreuses cours réelles ${ }^{28}$, et que l'art de la persuasion était devenu un devoir moral pour tous ceux qui voulaient s'impliquer dans la défense de la civitas.

Inversement, comme nous avons rappelé brièvement le contexte historique et vérifié la précision des éléments descriptifs qui jalonnent cette chanson, nous devons également nuancer ce qu'affirme Achille Tartaro. D'après lui, Guittone utilise " un langage figuré qui évite toute détermination de faits ou de circonstances", si bien que les différentes situations qui ont provoqué les invectives du poète contre la politique des gibelins de Florence " perdent toute caractéristique contingente ou occasionnelle pour assumer la valeur de situations emblématiques, exemplaires". Or, si Guittone "récupère le raffinement artistique de la poésie d'amour, de la poésie de style tragique " ${ }^{29}$, il le dépasse en rétablissant le lien entre poésie et actualité politique que les Siciliens avaient abandonné. La chanson Ahi dolze e gaia terra fiorentina, déjà citée pour l'image de la fleur, montre en revanche que Chiaro Davanzati n'exprime pas le même degré d'engagement éthique et politique que son modèle; la comparaison entre les deux compositions 
révèle aussi toure la force expressive qui caractérise les vers de Guittone... et bientôt ceux de Dante ${ }^{30}$.

Nous pouvons en conclure que Guitrone participe pleinement a la culture de son temps et qu'il manie parfaitement l'éloquence à des fins civiles, selon une vision politique déjà plus large que le seul cadre municipal : la défense de la Raison héritée de l'Antiquité suppose en effet que le regard se porte sur un idéal universel de vie en société enfin libérée des guerres intestines. Paradoxalement, les talents de Guittone que Dante s'acharnait à lui contester trouvent peut-être leur confirmation au chant $X$ de l'Enfer, si on émet l'hypothèse que l'idée d'attribuer des "remords "à Farinata pourrait lui être venue à la lecture de cette fameuse chanson de Montaperti.

Cécile LE LAY 


\section{Annexe}

Guittone D'Arezzo, Canzone di Montaperti (xIX) ${ }^{31}$

Ahi lasso, or è stagion de doler tanto

a ciascun om che ben ama Ragione,

ch'eo meraviglio u' trova guerigione,

ca morto no l'ha già corrotto e pianto,

vedendo l'alta Fior sempre granata

e l'onorato antico uso romano

ch'a certo pèr, crudel forte villano,

s'avaccio ella no è ricoverata :

ché l'onorata sua ricca grandezza

e 'l pregio quasi è già turto perito

e lo valor e 'l poder si desvia.

Oh lasso, or quale dia

fu mai tanto crudel dannaggio audito ?

Deo, com'hailo sofrito,

deritto pèra e torto entri 'n altezza?

Altezza tanta êlla sfiorata Fiore

fo, mentre ver' se stessa era leale, che ritenëa modo imperïale, acquistando per suo alto valore,

provinci' e terre, press'o lunge, mante ;

e sembrava che far volesse impero

sì como Roma già fece, e leggero

li era, c'alcun no i potea star avante.

E ciò li stava ben certo a ragione, 
GuITtONe D'AREZZO, Chanson de Montaperti (XIX)

Hélas! le temps du grand deuil est venu pour tous ceux qui sont épris du bon droit, si bien que je me demande où ils trouvent le remède pour que douleur et pleurs ne les aient pas déjà tués,

à la vue de la noble Fleur de tous temps porreuse de fruits 5 et de ses anciens us romains dignes d'honneur qui périront sans nul doute (vile et féroce cruauté !), si on ne la secourt pas au plus tôt :

car sa grandeur riche et honorée et son renom sont déjà presque anéantis, et sa vaillance et sa puissance se dévoient. Hélas! Quel jour jamais a-t-on ouï parler d'un si cruel dommage ? Dieu ! Comment as-tu souffert que la justice périsse et l'iniquité triomphe?

Si grande puissance dans cette Fleur défleurie régna, tant qu'elle était fidèle à elle-même et conservait l'usage impérial, en acquérant par sa noble vaillance maintes provinces et villes, proches ou lointaines; et il semblait qu'elle voulût bâtir un empire comme le fit jadis Rome, et cela lui était facile, car personne ne pouvait la surpasser.

Et il était certes juste qu'il en fût ainsi, 
ché non se ne penava per pro tanto,

como per ritener giustizi' e poso ;

e poi folli amoroso

de fare ciò, si trasse avante tanto,

ch'al mondo no ha canto

u' non sonasse il pregio del Leone.

Leone, lasso, or no è, ch'eo li veo tratto l'onghie e li denti e lo valore, e 'I gran lignaggio suo mort'a dolore, ed en crudel pregio[n] mis' a gran reo.

E ciò li ha fatto chi? Quelli che sono

de la schiatta gentil sua stratti e nati, che fun per lui cresciuti e avanzati sovra tutti altri, e collocati a bono;

e per la grande altezza ove li mise ennantîr sì, che 'l piagâr quasi a morte ; ma Deo di guerigion feceli dono, ed el fe' lor perdono;

e anche el refedier poi, ma fu forte e perdonò lor morte :

or hanno lui e soie membre conquise.

Conquis'è l'alto Comun fiorentino, e col senese in tal modo ha cangiato, che tutta l'onta e 'I danno che dato li ha sempre, como sa ciascun latino,

li rende, e i tolle il pro e l'onor tutto:

ché Montalcino av' abattuto a forza, Montepulciano miso en sua forza, e de Maremma ha la cervia e 'I frutto; 
car elle cuvrait moins pour son profit

que pour maintenir la justice et la paix ;

du jour où il lui plut

d'agir ainsi, elle s'étendit tant

qu'au monde il n'est de lieu

où ne retentissait la renommée du Lion.

Le Lion, hélas, n'est plus, car je vois qu'on lui a arraché les griffes et les dents et la vaillance, et que ses grandes familles ont été tuées à grande douleur, et mises en prison en toute injustice.

Et qui lui a fait cela ? Ceux-là mêmes issus et nés de sa noble race, qui furent nourris par lui, élevés au-dessus des autres et hissés à la place d'honneur;

et, grâce à cette grande hauteur où il les mit, s'enorgueillirent jusqu'à le blesser presque à mort ;

mais Dieu lui accorda la guérison, et lui leur accorda son pardon ; puis ils le blessèrent de nouveau, mais il montra sa force et leur fit grâce :

à présent ils l'ont abattu, lui et ses membres.

Elle est abattue la grande Commune de Florence, et elle a si bien fait l'échange avec Sienne que toute la honte et le dommage qu'elle lui a toujours causés, comme le sait tout Italien, lui sont rendus, et tout bien et honneur enlevés : car Sienne a détruit Montalcino par la force, mis Montepulciano sous son joug, et pris les cerfs et les fruits de Maremme ; 
Sangimignan, Pog[g]iboniz' e Colle

e Volterra e 'l paiese a suo tene ;

e la campana, le 'nsegne e li arnesi

e li onor tutti presi

ave con ciò che seco avea di bene.

E tutto ciò li avene

per quella schiatta che più ch'altra è folle.

Foll'è chi fugge il suo prode e cher danno,

e l'onor suo fa che vergogna i torna,

e di bona libertà, ove soggiorna

a gran piacer, s'aduce a suo gran danno

sotto signoria fella e malvagia,

e suo signor fa suo grand'enemico.

A voi che siete ora in Fiorenza dico, che ciò ch'è divenuro, par, v'adagia ;

e poi che li Alamanni in casa avete,

servite. $i$ bene, e faitevo mostrare

le spade lor, con che v'han fesso i visi,

padri e figliuoli aucisi ;

e piacemi che lor dobiate dare, perch'ebber en ciò fare

fatica assai, de vostre gran monete.

Monete mante e gran gioi' presentate ai Conti e a li Uberti e alli altri tutti ch'a tanto grande onor v'hano condutti, che miso v'hano Sena in podestate;

Pistoia e Colle e Volterra fanno ora

guardar vostre castella a loro spese ;

e 'I Conte Rosso ha Maremma e 'l paiese,

Montalcin sta sigur senza le mura ; 
San Gimignano, Poggibonsi, Colle, Volterra et ses terres, elle les a fait siens ;

la cloche, les enseignes, les engins,

et les honneurs, elle les a tous pris

et tous les biens rassemblés en ce lieu.

Et tout cela advient à cause

de cette engeance plus que toute autre folle.

Fol est qui fuit son bien et cherche son dommage, et fait que son honneur se tourne en honte, et, s'écartant de la bonne liberté dont il jouit avec grand plaisir, se soumet pour son grand malheur

à une seigneurie félonne et mauvaise, et prend pour seigneur son pire ennemi. $A$ vous qui êtes à présent à Florence, je dis que ce qui s'est passé vous agrée, semble-t-il ;

et puisque les Allemands sont chez vous, servez-les bien, et faites-vous montrer

leurs épées avec lesquelles ils ont balafré vos visages, et tué vos parents et vos enfants ;

et j'aimerais que vous leur donniez

- puisque cela leur coûta

bien de la peine - vos pièces d'or en grande quantité.

Offrez donc maintes pièces et force joyaux aux Conti, aux Uberti, et à tous ceux qui vous ont conduits à un si grand honneur qu'ils ont mis Sienne en votre pouvoir,

que Pistoia, Colle et Volterra font à présent garder vos châteaux à leurs frais, et le Comte Rosso a Maremme et ses terres, Montalcino est en sécurité sans ses remparts, 
de Ripafratta temor ha 'I pisano,

e 'l perogin che 'l lago no i tolliate,

e Roma vol con voi far compagnia.

Onor e segnoria

adunque par e che ben tutto abbiate:

ciò che desïavate

potete far, cioè re del toscano.

Baron lombardi e romani e pugliesi

e toschi e romagnuoli e marchigiani,

Fiorenza, fior che sempre rinovella,

a sua corte v'apella,

che far vol de sé rei dei Toscani,

dapoi che li Alamani

ave conquisi per forza e i Senesi. 
de Ripafratta se méfie le Pisan,

et le Pérousin craint que vous ne lui preniez le lac,

et Rome veut s'allier avec vous !

Honneur et seigneurie

sont donc à vous, semble-t-il, ainsi que tous les biens :

ce que vous souhaitiez,

vous pouvez le faire, à savoir être rois de Toscane.

Barons de Lombardie et de Rome et des Pouilles

et de Toscane et de Romagne et des Marches,

Florence, fleur qui toujours se renouvelle,

à sa cour vous appelle

car elle veut devenir le roi des Toscans

95

après que les Allemands

elle les a abattus par la force, et les Siennois. 


\section{Cécile Le LAY}

\section{Notes}

1. Cetre poésie fait partie des textes publiés par Gianfranco Contini : Poeti del Duecento, Milano-Napoli, Ricciardi, 1960, r. I, p. 206-209.

2. Notre étude se réfère à plusieurs articles publiés in : Guittone d'Arezzo nel settimo centenario della morte. Arti del Convegno Internazionale di Arezzo 22-24 aprile 1994, éd. par Michelangelo Picone, Firenze, Cesati, 1995 , ainsi qu'à la fondamentale monographie de Claude Margueron : Recherches sur Guittone d'Arezzo, Paris, Presses Universitaires de France, 1966.

3. G. CONTINI, Poeti del Duecento..., t. I, p. 414-416.

4. Brunetro Latini, ayant appris la catastrophe de Montaperti alors qu'il revenait d'une ambassade en Espagne pour le compte de Florence, préféra s'exiler en France : il jouera un rôle politique fondamental à son retour, après la victoire guelfe de Bénévent (1266).

5. Cf. Giovanni VILLANI, Cronica, VI-VII et Robert DAVIDSOHN, Storia di Firenze, Firenze, Sansoni, 1972, vol. II, Guelfi e Ghibellini, parte I, Lotte sveve.

6. L'inscription, très longue, affirme notamment à propos de Florence : "tamquam Roma sedet semper ductura triumphos".

7. Traduction française de Marc Scialom, in DANTE, CEuvres complètes, Paris, La Pochotèque, 1996, Enfer, X, 85-86, p. 638. Texte original (selon la Società Dantesca Italiana) : "Io strazio e il grande scempio// che fece l'Arbia colorata in rosso". 


\section{LE DÉSASTRE DE MONTAPERTI}

8. Ruggero STEFANINI, "Guittone poeta politico ", in Guittone d'Arezzo..., p. 170.

9. Nous réservons pour une prochaine étude l'approfondissement de la comparaison entre ces deux compositions (cf. GUITTONE D'AREZZO, Lettere, éd. par Claude Margueron, Bologna, Commissione per i Testi di Lingua, 1990, p. 155-179 : Lettre XIV, avec introduction, bibliographie et ample commentaire, notamment sur les liens avec la chanson de Montaperti).

10. Chiaro Davanzati, Ahi dolze e gaia terra fiorentina, v. 3 : "Fior de l'altre, Fiorenza "; v. 20 : “fior de la gente »; v. 43 : “Fiorenza non pos' dire, che se' sfiorita ".

11. Dante ALIGHIERI, De vulgari eloquentia (II vi 5) : "Eiecta maxima parte florum de sinu tuo, Florentia [...]"

12. Pour une présentation plus approfondie des différents sens du terme ragione et de ses occurrences chez Guittone, en comparaison avec certains textes de la pensée politique et juridique de l'époque, voir notre thèse de doctorat : Cécile LE LAY, Le Droit et la Justice dans la littérature italienne du XIIF siecle: Guittone d'Arezzo et Jacopone da Todi, Université Paris III - Sorbonne nouvelle, novembre 2004 , p. 227 et s.

13. Claude MARGUERON, Recherches..., p. 54.

14. Claude Margueron cite plusieurs études qui ont prouvé que le sens de la continuité entre la ville antique de Rome et la ville moderne de Florence était bien vivant dans la conscience des Toscans du XIII ${ }^{e}$ siècle : GUITTONE D'AREZZO, Lettere..., p. 170.

15. "Cittade èe uno raunamento di gente fatta per vivere a ragione ; onde non sono detri cittadini d'uno medesimo comune perché 


\section{Cécile Le Lay}

siano insieme accolti dentro ad uno muro, ma quelli che insieme sono accolti a vivere ad una ragione [...]. Et là dove dice che non manteneano ragione intendo " ragione " cioc̀ giustizia, della quale dicono i libbri della legge che giustizia è perpetua e ferma volontade d'animo che dae a ciascuno sua ragione ": Brunetto LATINI, La Rettorica, Testo critico di Francesco Maggini, Prefazione di Cesare Segre, Firenze, Le Monnier, 1968, \$2. 4 p. 13 et $\$ 2.9$ p. 20.

16. G. CONTINI, Poeti del Duecento..., t. I, p. 200-205.

17. "Membrar noia anche me fae/como bon uso e ragione/n'è partuto e rea condizione/e torto e falsezza li stae " (XV, 29-32), ibid., t. I, p. 200-205.

18. Ibid., t. I, p. 214-217.

19. Francesco MAZZONI, "Tematiche politiche fra Guittone e Dante ", in Guittone d'Arezzo..., p. 366.

20. Ruggero STEFANINI, Guittone poeta politico..., p. 170.

21. "E tutto ciò li avene/per quella schiatta che più ch'altra è folle.// Foll'è chi fugge il suo prode e cher danno,/e l'onor suo fa che vergogna i torna " (XIX, 59-62).

22. Si Dante place les traîtres au fond de l'Enfer, cela prouve qu'à l'époque comme aujourd'hui la trahison était considérée comme l'acte criminel le plus grave.

23. "Ond'io non so nessuno/ch'io volesse vedere/la mia cittade avere/del tutto a la sua guisa,/né che fosse in divisa ;/ma tutti per comune/tirassero una fune/di pace e di ben fare,/ché già non può scampare/terra rotta di parte" : Brunetto LATINI, "Il Tesoretto ", v. 170-179, in G. CONTINI, Poeti del Duecento..., t. II, p. 182. 


\section{LE DESASTRE DE MONTAPERTI}

24. Paolo CHERCHI, "Lepifrasi in Guittone", in Guittone d'Arezzo..., p. 33-34 et 45. Il donne cet exemple d'épiphrase (XIX, 96-97) en appendice (p. 46).

25. En s'appuyant sur Ernst Robert CURTIUS (La littérature européenne et le Moyen Âge latin, Paris, Presses Universitaires de France, 1956), Claude Margueron rappelle (en se référant à Brunetto Latini : Tres., III X, 1 et Tes., VIII 10, 42) que «l'Antiquité et le Moyen Âge voyaient dans la poésie et l'éloquence deux aspects d'une même technique et non deux activités littéraires radicalement différentes " (Claude MARGUERON, Recherches..., p. 365).

26. Ruggero STEFANINI, Guittone poeta politico.... p. 170.

27. Ibid., p. 171. Larticle est très intéressant pour les comparaisons entre Guittone et Dante, et pour les nombreuses observations stylistiques, mais nous préférons la prudence d'interprétation de Claude Margueron à propos de L'attitude politique de Guittone (in Claude MARGUERON, Recherches..., p. 49-66).

28. Cf. C. MARGUERON, Recherches..., Troisième partie : "Les relations littéraires et politiques de Guittone ", Chapitre II : "Amis et connaissances", p. 143-270 ; et GUITTONE D'AREZZO, Canzoniere. I sonetti d'amore del codice Laurenziano, éd. par Lino Leonardi, Torino, Einaudi, 1994, p. XIII-LIX.

29. Achille TARTARO, Il manifesto di Guittone e altri studi fra Due e Quattrocento, Roma, Bulzoni, 1974, p. 21-22.

30. Pour de plus amples détails sur cette comparaison, voir Ruggero STEFANINI, Guittone poeta politico..., p. 174-176.

31. Cette poésie fait partie du recueil : Poeti del Duecento, éd. par G. Contini, Milano-Napoli, Ricciardi, $1960\left(1995^{2}\right)$, vol. 2, t. 1 , p. 20-23 (vol. I, t. I, p. 206-209). 